\title{
NOUVELle
}

\section{Pore nucléaire et organisation fonctionnelle de la chromatine}

Emmanuelle Fabre
Unité de Génétique Moléculaire des Levures, URA 2171 CNRS, UFR

927, Université Pierre et Marie Curie,

Département de Génomes et Génétique, Institut Pasteur,

25, rue du Docteur Roux, 75724 Paris Cedex, France.

efabre@pasteur.fr
$>$ L'organisation d'un noyau et des chromosomes qui s'y trouvent confinés n'est pas aléatoire et l'incidence fonctionnelle de cette organisation sur la chromatine est importante. Non seulement pour la réplication de l'information génétique et sa transmission, mais aussi pour la transcription de cette information et comme cela sera développé ici, pour le mode de réparation de l'ADN, en cas de dommages.

De nombreuses études sur ce sujet ont utilisé la levure de boulanger (Saccharomyces cerevisiae) comme modèle. Cet organisme eucaryote unicellulaire, facile à manipuler génétiquement, montre que beaucoup des principes qui y sont découverts se vérifient dans des organismes plus complexes. Ainsi les extrémités des 16 chromosomes linéaires de $S$. cerevisiae sont organisées en périphérie du noyau à proximité de l'enveloppe nucléaire. Une telle organisation peut se retrouver à des degrés divers dans certains types de cellules de métazoaires. Les 32 télomères de S. cerevisiae sont de plus regroupés en foyers (environ 5-8 structures discrètes) [1]. La nature de l'association préférentielle observée entre ces télomères n'est pas connue et la dynamique d'un télomère par rapport à ces foyers ne l'est pas plus.

$\varepsilon$ n revanche, des études récentes ont mis en évidence la nature des liens responsables de l'ancrage des télomères à la périphérie nucléaire. Deux voies non-redondantes ont été découvertes, celle qui utilise l'hétérodimère Ku70/Ku80 affin pour les extrémités ADN et celle qui emploie Sir4p. Cette dernière, avec Sir2p et Sir3p (Sir : silent information regulator), est enrichie en périphérie du noyau et participe à l'établissement de la chromatine silencieuse trouvée à la périphérie nucléaire. Un partenaire privilégié de Sir4p, Esclp, sert de lien entre Sir4p et l'enveloppe nucléaire [2].

Ces protéines ne possédant pas de séquence qui pourrait formellement les ancrer à la membrane, c'est ici qu'entre en jeu le pore nucléaire. C'est à travers ce complexe macromoléculaire hautement organisé et conservé, inséré dans la double enveloppe nucléaire, que se font les nombreux échanges de molécules entre noyau et cytoplasme. Au sein du pore nucléaire, on distingue le sous-complexe Nup84 (Nup107 chez les métazoaires) composé de 7 nucléoporines [3]. In vitro, Nup107 est le premier complexe à pouvoir s'associer à la chromatine après la mitose. Cette association est cruciale pour reformer l'enveloppe nucléaire (dispersée lors de la mitose chez les métazoaires) [4]. La notion selon laquelle le pore nucléaire n'aurait pas comme seule fonction de permettre le transport nucléo-cytoplasmique mais jouerait aussi un rôle dans l'architecture du noyau, en permettant l'ancrage de la chromatine, commence à se dessiner.

Et chez la levure ? En étudiant la localisation tridimensionnelle de l'extrémité d'un chromosome marqué de façon fluorescente dans des cellules vivantes, nous avons montré que des mutants du complexe Nup84 en affectent sa localisation, à un degré comparable à celui des mutants ku70 et sir4 [5]. II est à noter que dans les conditions choisies pour cette étude, le transport noyau-cytoplasme n'est pas affecté. Des mutations dans ce complexe pourraient aussi partiellement désorganiser les foyers télomériques [6]. Ainsi, l'organisation de la chromatine en périphérie du noyau nécessite un pore nucléaire fonctionnel qui pourrait fournir un des liens nécessaires à l'ancrage de ku70 ou sir4/escl [2].

L'incidence fonctionnelle d'une telle désorganisation chromosomique se manifeste sur l'état transcriptionnel de la chromatine. Les régions proches des extrémités télomériques (subtélomères) sont transcriptionnel- lement inertes et ressemblent par certains critères à l'hétérochromatine des eucaryotes plus complexes [7]. Si le complexe Nup84 n'est pas fonctionnel, l'extrémité télomérique délocalisée devient transcriptionnellement active [5]. En se délocalisant, il est possible que le chromosome échappe à la concentration périphérique des protéines Sir; la délocalisation partielle de la protéine Sir3p pourrait aussi expliquer l'effet observé sur la transcription [5].

De façon paradoxale, il a été récemment montré que le pore nucléaire joue aussi un rôle protecteur vis-à-vis de la répression transcriptionnelle. D'abord parce qu'un ensemble de nucléoporines (mais non celles du complexe Nup84) interagissent avec les gènes actifs ; c'est au pore nucléaire que des gènes activés peuvent être transitoirement trouvés; une protéine mobile du pore, Nup2p, pourrait également marquer les régions à protéger de l'inactivation transcriptionnelle (références dans [8]). Comprendre comment se fait le partage de domaines chromatiniens actifs et inactifs en périphérie nucléaire et comment le pore nucléaire y participe reste un défi à relever (Figure 1).

Les conséquences du complexe Nup84 non fonctionnel s'étendent au-delà de la transcription. Nous avons déterminé que ce défaut modifiait la capacité à réparer une coupure double brins (CDB), lorsque celle-ci avait lieu dans les subtélomères [5]. Cette observation découle d'études qui utilisent l'endonucléase I-Scel pour engendrer des CDB à des endroits précis du génome nucléaire [9]. Toute CDB non réparée est délétère pour la cellule, y compris celle qui est produite par I-Scel. Des machineries de surveillance et de réparation existent; ces dernières font intervenir la recombinaison homologue ou, en l'absence d'homologie, le raboutage par ligature des bouts d'ADN non homologues (NHEJ, non homologous end joining). En provoquant des $C D B$ à différentes positions le 
long d'un chromosome, il a été montré qu'en fonction de celles-ci, le mode de réparation variait [9]. En région centrale, la majorité des cellules ne survivent pas à la CDB et seul le NHEJ est utilisé comme moyen de réparation; dans les subtélomères, d'autres évènements peuvent prendre place, ce qui se traduit par une survie plus élevée à la CDB [9]. Or, dans les mutants du complexe Nup84, la capacité à réparer une CDB subtélomérique est affectée, parfois au point de ramener la survie après CDB dans un subtélomère au niveau de celle qui est observée en région centrale. Curieusement ce phénomène ne touche que le subtélomère. Est-ce dû à sa délocalisation? À des modifications de la chromatine comme le manifeste la perte de la répression transcriptionnelle? Une partie de la réponse provient de l'étude du mutant escl.
Dans ce mutant, dont nous avons vérifié l'incapacité à ancrer l'extrémité du chromosome, la répression transcriptionnelle est maintenue, mais, à l'inverse, la capacité de survivre à une $\mathrm{CDB}$ subtélomérique est réduite [5]. En dissociant l'effet sur la transcription de celui sur la réparation, on favorise l'hypothèse selon laquelle c'est un positionnement périphérique qui faciliterait la réparation.

Le complexe Nup84 a déjà été impliqué dans des phénomènes de réparation de I'ADN; dans des cribles affectant la résistance à des dommages de l'ADN, et dans des interactions génétiques avec des gènes engagés dans la réplication ou la réparation [10]. C'est la première fois cependant qu'une spécificité de réparation est montrée dans un

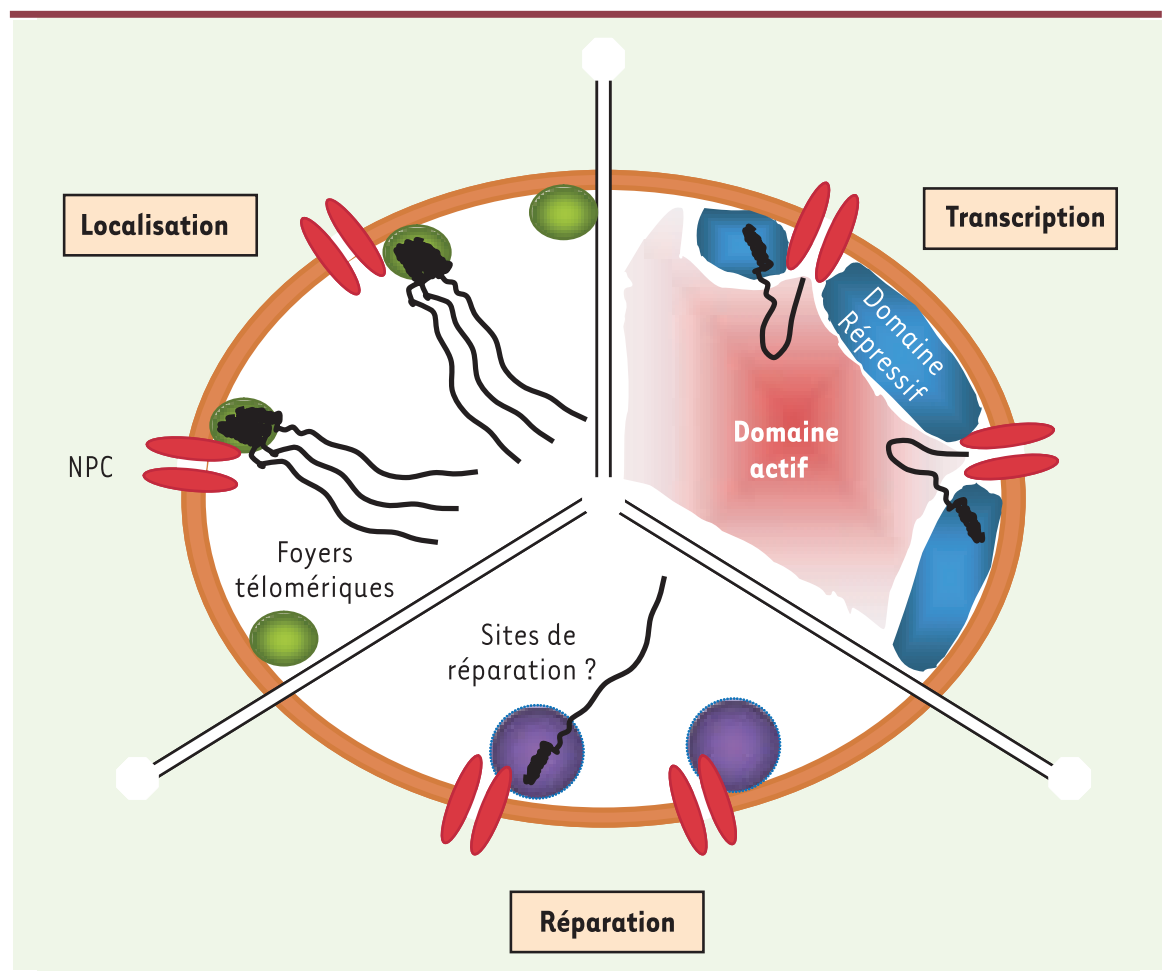

Figure 1. Noyau de levure schématisé. La double enveloppe nucléaire (orange) sépare nucléoplasme (intérieur) du cytoplasme (extérieur). Les pores nucléaires (NPC, en rouge) traversent la double enveloppe nucléaire. Le complexe Nup84 fait partie intégrante du NPC. Le schéma est arbitrairement séparé en trois domaines fonctionnels (localisation, transcription, réparation). Localisation : les foyers télomériques (en vert) regroupent plusieurs (sub)-télomères (ressort noir) à l'extrémité des chromosomes linéaires de levure (trait noir). Ku70/ku80 se fixe aux télomères. Les protéines Sir sont concentrées dans ces foyers (voir texte pour détails). Le complexe Nup84 participe à cette localisation. Transcription: les NPC départagent les domaines périnucléaires répressifs pour la transcription (en bleu), des domaines actifs pour la transcription (en rouge). Réparation : des sites de réparation (en violet) pourraient se trouver à proximité des NPC et favoriser la réparation des régions subtélomériques.

domaine particulier d'un chromosome. Malgré la possibilité d'échanges entre subtélomères, la recombinaison entre ceux-ci n'est pas très efficace [9], nos résultats montrent que l'ancrage à la périphérie le favorise; il est possible que le complexe Nup84 permette le positionnement de protéines de réparation favorisant ainsi la réparation des séquences d'ADN endommagées à proximité, mais aussi que des systèmes de surveillance ou de réparation spécifiques existent aux subtélomères. II reste beaucoup à comprendre en ce qui concerne les liens entre pore nucléaire et réparation de l'ADN, mais il apparaît que l'organisation de la chromatine à l'extrémité des chromosomes semble importante pour assurer leur intégrité. $\diamond$

Nuclear pore and functional

organization of chromatin

\section{REMERCIEMENTS}

Ce travail a bénéficié d'un financement de l'ARC ( $\left.n^{\circ} 3266\right)$. Je remercie Bertrand Llorente pour sa lecture critique de ce texte.

\section{RÉFÉRENCES}

1. Gotta M, Laroche T, Formenton A, et al. The clustering of telomeres and colocalization with Rapl, Sir3, and Sir4 proteins in wild-type Saccharomyces cerevisiae. J Cell Biol1996; 134: 1349-63.

2. Taddei A, Hediger F, Neumann FR, et al. Separation of silencing from perinuclear anchoring functions in yeast Ku80, Sir4 and Escl proteins. EMBO J2004; 23 : 1301-12.

3. Teixeira MT, Siniossoglou S, Podtelejnikov S, et al. Two functionally distinct domains generated by in vivo cleavage of Nup145p : a novel biogenesis pathway for nucleoporins. EMBOJ 1997 ; 16 : 5086-97.

4. Walther TC, Alves A, Pickersgill $H$, et al. The conserved Nup 107-160 complex is critical for nuclear pore complex assembly. Cell 2003; 113: 195-206.

5. Therizols P, Fairhead C, Cabal GG, et al. Telomere tethering at the nuclear periphery is essential for efficient DNA double strand break repair in subtelomeric region. J Cell Biol 2006 ; 172: 189-99.

6. Galy V, Olivo-Marin JC, Scherthan H, et al. Nuclear pore complexes in the organization of silent telomeric chromatin. Nature 2000 ; 403 : 108-12.

7. Pryde $F E$, Louis $\varepsilon$ J. Limitations of silencing at native yeast telomeres. EMBO J 1999 ; $18: 2538-50$.

8. Dilworth DJ, Tackett AJ, Rogers RS, et al. The mobile nucleoporin Nup2p and chromatin-bound Prp20p function in endogenous NPC-mediated transcriptional control. J Cell Biol2005; 171 : 955-65.

9. Ricchetti M, Dujon B, Fairhead C. Distance from the chromosome end determines the efficiency of double strand break repair in subtelomeres of haploid yeast. J Mol Biol 2003; 328: 847-62.

10. Loeillet S, Palancade B, Cartron M, et al. Genetic network interactions among replication, repair and nuclear pore deficiencies in yeast. DNA Repair (Amst) 2005; 4: 459-68. 